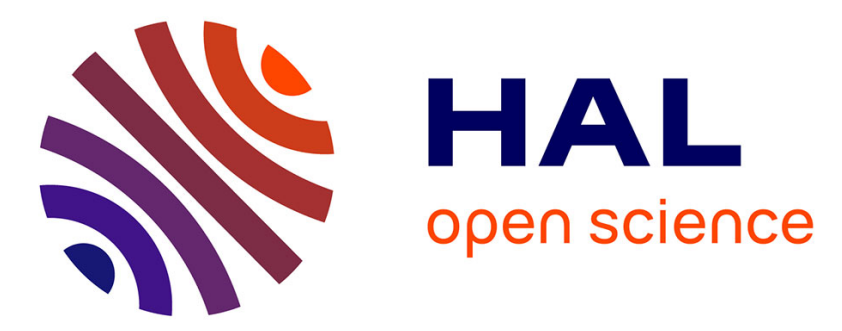

\title{
La littérature comme instrument de sauvegarde du patrimoine culturel immatériel des Kurdes de Turquie
}

Sylvain Cavaillès

\section{To cite this version:}

Sylvain Cavaillès. La littérature comme instrument de sauvegarde du patrimoine culturel immatériel des Kurdes de Turquie. Anatoli, 2015, Patrimoines culturels et fait minoritaire en Turquie et dans les Balkans, 6. hal-01226718

\section{HAL Id: hal-01226718 \\ https://hal.science/hal-01226718}

Submitted on 10 Nov 2015

HAL is a multi-disciplinary open access archive for the deposit and dissemination of scientific research documents, whether they are published or not. The documents may come from teaching and research institutions in France or abroad, or from public or private research centers.
L'archive ouverte pluridisciplinaire HAL, est destinée au dépôt et à la diffusion de documents scientifiques de niveau recherche, publiés ou non, émanant des établissements d'enseignement et de recherche français ou étrangers, des laboratoires publics ou privés. 


\title{
La littérature comme instrument de sauvegarde du patrimoine culturel immatériel des Kurdes de Turquie
}

Sylvain Cavaillès

\begin{abstract}
«Est-ce que vous n'avez jamais étudié l'histoire ? Il n'y a ni Kurdes ni Kurdistan en Turquie. (...) Il n'y a pas de peuple nommé le «peuple kurde ». Ils sont tous Turcs. »
\end{abstract}

Cemal Gürsel ${ }^{1}$

\begin{abstract}
C'est contre le négationnisme, dont cette déclaration du $4^{\mathrm{e}}$ président de la République de Turquie n'est qu'une expression-type, et contre tout ce qu'il a impliqué pendant des décennies, qu'a dû être préservée au cours du $\mathrm{xx}^{\mathrm{e}}$ siècle l'identité culturelle et se construire la littérature moderne des Kurdes de Turquie. La division du Kurdistan au lendemain du traité de Lausanne, les politiques répressives de l'État kémaliste visant, à la suite des révoltes des années 20 et 30, à la turquification des populations minoritaires de l'Est anatolien, la «thèse turque de l'histoire » et la «théorie de la langue-soleil » qui affirmait l'origine turque de toutes les langues et de toutes les civilisations du monde auront réussi à maintenir jusqu'à une époque récente une irrépressible chape de plomb sur l'expression de l'identité kurde, s'attaquant à l'outil de sa possibilité même, la langue, dont l'interdiction commence tout juste à être levée. C'est donc, comme nous allons le voir, hors de Turquie, en diaspora, que cette identité a pu être préservée, cette mémoire sauvée de l'oubli et cultivée.

Alors que leur statut de peuple sans État empêche jusqu'à la reconnaissance de leur patrimoine culturel par l'UNESCO - si ce n'est, et dans une très faible mesure, par procuration, par le truchement des ponts interculturels existant dans la région (on trouve inscrits au patrimoine culturel immatériel de l'humanité la musique des bakhshis du Khorasan ou encore le Newroz) la sauvegarde de la mémoire collective et de l'identité culturelle des Kurdes,
\end{abstract}

\footnotetext{
1. Cemal Gürsel, président de la République de Turquie de mai 1960 à mars 1966, cité par J. Nebez, Kurdistan und seine Revolution, Berlin, 1972, p. 80 (BozARSLAN H., "Le kémalisme et le problème kurde... », 1992, p. 73).
} 
le champ politique étant fermé, ne pouvait se faire que dans le champ intellectuel ; en l'occurrence, la langue étant la cible première par laquelle devait se faire l'assimilation, le champ linguistique et son corollaire naturel, la littérature.

Lorsque l'on aborde la littérature kurde, il convient d'évoquer trois périodes : la littérature classique, la littérature dite diasporique ou de l'exil et la littérature contemporaine. La littérature classique (ainsi que les différentes formes de littérature orale) ayant souvent été traitée, nous n'en ferons qu'un rapide survol. Nous ferons ensuite un tour d'horizon des organes de presse publiés au début du siècle à Istanbul par les élites intellectuelles kurdes et notamment le clan des Bedir Khan, qui intéresse notre sujet en ce que cette histoire intellectuelle, liée au mouvement nationaliste kurde, a été et continue d'être revendiquée en tant que patrimoine commun, et en ce qu'elle prélude à la renaissance linguistique et littéraire qui ne commencera à se réaliser qu'en diaspora, d'abord en Syrie sous mandat français dans les années 1930 avec la revue Hawar, puis au début des années 1980 en Suède, notamment par l'écrivain Mehmed Uzun, considéré aujourd'hui comme le pionnier du roman kurde contemporain, sur lequel nous concentrerons notre attention avant d'évoquer le travail de patrimonialisation de certaines éditeurs de littérature kurde.

\section{L’héritage}

Kendal Nezan le souligne dans sa préface à l'édition française de l'épopée kurde Mamé Alan établie par Roger Lescot, les Kurdes ont produit au cours des siècles une littérature d'une grande richesse. Si la littérature orale a pu continuer de se transmettre dans les époques les plus troublées, le patrimoine écrit a dramatiquement souffert dans les années 1920 et 1930 lorsque, poussée à la répression par les révoltes de l'Est anatolien, la jeune République de Turquie a entamé sa politique de négation de l'identité kurde, occasionnant des pertes irrémédiables, notamment en incendiant les bibliothèques des medersas et des mosquées.

Selon Alaeddin Seccadî, les premiers écrits kurdes dateraient du viI ${ }^{\mathrm{e}}$ siècle. La littérature kurde aurait donné ses prémices entre le $\mathrm{x}^{\mathrm{e}}$ et le $\mathrm{XII}^{\mathrm{e}}$ siècles, avant de véritablement éclore à partir des $\mathrm{Xv}^{\mathrm{e}}-\mathrm{XvI}^{\mathrm{e}}$ siècles. Cette littérature que nous dirons classique est une littérature de cour, produite par des poètes mystiques, érudits, issus des medersas des différentes principautés du Kurdistan de l'époque, connaissant l'arabe, le persan et le turc mais préférant écrire en 
kurde. Ainsi de Melayê Cizîrî² (1570-1640), poète de la cour du Botan, auteur d'un Divan publié pour la première fois en 1904 à Berlin par l'orientaliste allemand Martin Hartman ${ }^{3}$, en kurde en 1987 par les éditions Roja $\mathrm{Nu}^{4}$ de Stockholm, puis en turc en 20075. Son disciple Feqiyê Teyran (1590-1660) est l'auteur de trois ouvrages considérés comme des classiques de la littérature kurde : Şêxê Senan [Le cheikh de Senan], Qiseya Bersiyayî [L'histoire de Bersiya] et Qewlê Hespê Reş [Le conte du cheval noir]. D'abord respectueux des enseignements et du style de son maître, il a fini par forger son propre style, se rapprochant de la langue populaire et s'éloignant de l'inspiration mystique $^{6}$. Viennent ensuite le pilier de la littérature kurde, le poète « national » Ehmedê Xanî (1651-1707) et son chef d'œuvre, Mem û Zîn [Mam et Zin], inspiré d'une pièce maîtresse de la littérature orale, l'épopée Mame Alan. Xanî s'y fait le chantre du sentiment national kurde, prônant l'union des Kurdes contre leurs oppresseurs et la constitution d'un État autour d'une langue et d'une culture communes. Nikitine n'est pas le seul à insister sur ses qualités de poète et il ne se prive ni de comparer son œuvre au Divan de Rumi, ni de rappeler qu'il a également écrit quelques textes sur l'art poétique, dont le premier dictionnaire des rimes et de la métrique de la poésie kurde. Mem $\hat{u}$ Zîn, en tant que pièce maîtresse d'un patrimoine littéraire, fait l'objet de nombreuses éditions, mais aussi d'adaptations théâtrales ou de réécritures. En 2014 le théâtre municipal de Diyarbakır a présenté une mise en scène de ce texte, d'après une adaptation en kurde moderne réalisée par le poète Kawa Nemir, qui a rencontré un grand succès public et est appelée à être reprise. D'autres adaptations ou réinterprétations littéraires de ce poème épique ont vu le jour ces dernières années, dont la plus remarquable est l'œuvre d'un jeune romancier de Diyarbakır, Erhan Sunar. Son livre Veda Oyunu, écrit en turc, fait justement le lien entre théâtre et roman en racontant l'histoire de Mem $\hat{u}$ Zîn du point de vue de jeunes acteurs préparant une mise en scène adaptée du texte de Xanî.

Si nous avons isolé ces trois noms, les plus célèbres de cette période classique, pour le rayonnement qu'ils ont acquis dès l'instant où le mouvement

2. Celui-ci n'est pas le premier poète kurde qui nous soit connu, Celadet Bedir Khan ayant révélé, dans le $n^{\circ} 33$ de la revue Hawar, l'existence d'un Divan d'un poète connu sous le nom d'Elî Herîrî, originaire de la province de Hakkari : cf. Uzun M., Destpekâ..., 1992, p. 22.

3. Nezan K., 1999, p. 11.

4. Mehmed Uzun mentionne deux autres éditions pré-républicaines, dont l'une à Istanbul en 1919 : Uzun M., Destpekâ..., 1992.

5. Traduit par Osman Tunç et publié par Kent Yayınları.

6. Uzun M., Destpekâ..., 1992. 
national kurde a saisi l'importance de la redécouverte du patrimoine littéraire, il ne faudrait pas les croire isolés dans leur époque. Pour un aperçu plus détaillé, on se réfèrera à la foisonnante anthologie de la poésie kurde de Selim Temo qui répertorie une cinquantaine d'auteurs avant Ehmedê Xanî.

Nous pourrions également mentionner ici deux textes dont la visée n'est pas poétique, mais qui ont une place au sein du patrimoine immatériel des Kurdes. Il s'agit des deux livres sacrés des Ézidis, le Kitab El-Cilwe [Livre de la révélation] et le Mishefa Reş [Livre noir]. Le premier a été rédigé au XII ${ }^{\mathrm{e}}$ siècle par le cheikh Adî, prophète de la religion ézidie qui figure dans l'anthologie de Temo en tant que poète. Les Ézidis, mentionnés en 1997 par Murathan Mungan, auteur turc d'ascendance arabo-kurde, dans son ouvrage autobiographique Paranin Cinleri [Les djinns de l'argent], connaissent depuis quelques années une certaine fortune éditoriale et sont redécouverts par les Kurdes comme faisant partie intégrante de leur communauté, leur culture, leur religion constituant une sorte de patrimoine partagé à l'intérieur du groupe minoritaire, alors même que les Ézidis eux-mêmes ont parfois tendance à réfuter cette filiation. L'actuelle épuration ethnique menée par DAECH met ces liens immatériels en lumière comme on voit les municipalités de Diyarbakır ou Batman accueillir les populations ézidies déplacée, ou l'université Artuklu de Mardin organiser sur le vif des conférences sur les drames vécus par les Ézidis à travers l'histoire, leur culture et la gestion de la crise actuelle, dans un contexte où le Sud-Est anatolien assiste au retour forcé d'une population chassée en dernier lieu par la répression de la guérilla kurde dans les années 1990. Notons que le recueil de Joyce Blau, Mémoire du Kurdistan intègre également « une prière yézidie ».

\section{L'enjeu patrimonial dans la presse kurde pré-républicaine}

Dès la fin du xIx ${ }^{\mathrm{e}}$ siècle, l'essor de la presse va permettre au mouvement national kurde de prendre conscience de l'importance de ces valeurs immatérielles que sont la langue, l'histoire et la littérature. Les périodiques publiés dès le Kurdistan de Mithat Bedir Khan sont confrontés à une double problématique de diffusion et d'éducation : les populations doivent être alphabétisées pour que l'héritage puisse être transmis. Les diverses initiatives éditoriales de la période pré-républicaine, outre le fait d'être souvent les organes d'associations politiques, ont en commun d'être, dans le domaine culturel, à la fois 
dans une démarche de sauvegarde, de découverte et d'établissement d'un patrimoine commun, dans lequel l'aspect linguistique a une place centrale.

La question du patrimoine immatériel des Kurdes se pose dès l'apparition du premier journal kurde, Kurdistan, publié le 22 avril $1898^{7}$ au Caire, puis à Genève et Folkestone, sous la houlette de Mikdat Mithat Bedir Khan, descendant du fameux émirat de Djézireh Botan. La création de ce journal en kurmanji est d'abord motivée, selon son fondateur, par la nécessité d'éduquer les populations kurdes en manque de culture, dépossédées de leur propre histoire et ne sachant ni lire ni écrire leur langue. L'ensemble des journaux et revues qui lui succèderont dans cette période qui prendra fin au lendemain de la Première Guerre mondiale auront en commun cette visée éducative qui passe par l'alphabétisation, la transmission des nouvelles du monde et la connaissance de l'histoire et de la littérature des Kurdes.

L'année 1908 voit la naissance à Istanbul de trois journaux. Le Kürt Teavün ve Terakki Gazetesi [Journal pour le développement et le progrès des Kurdes], organe de l'association du même nom, paraît de décembre 1908 à mars 1909; on lui connaît neuf numéros. Dirigée par Piremêrd, auteur de poésie, de nouvelles mais aussi de théâtre originaire de Süleymaniye, cette publication rédigée en turc et en kurde kurmanji ou sorani fait la part belle à la langue et à la littérature kurdes et prend position pour une éducation en langue maternelle, la création d'écoles et de bibliothèques. Le journal Şark ve Kürdistan [L'Orient et le Kurdistan], qui paraît à Istanbul en 1908, rédigé en turc et en kurde et dont la coloration est plus politique, se fait également l'écho de cette revendication. Un nouveau Kurdistan, publié par Ahmed Süreyya Bedir Khan, frère du fondateur du premier journal, paraît la même année et poursuivra sa publication jusqu'en 1913, puis 1917. Résolument anti-hamidien, le journal est porté par un espoir de liberté, de justice et par la foi dans le développement économique et culturel des populations kurdes. L'ottomanisme est présenté comme le remède miracle pour les minorités de l'Empire et notamment les Kurdes, qui doivent s'unir et se soutenir sous une même identité. On trouve dans le troisième numéro le texte d'une conférence prononcée par Abdurrahman Bedir Khan, où il évoque les heurts entre Kurdes et Arméniens en en reliant la cause au pouvoir hamidien, tout en prévoyant la fin prochaine des inimitiés, les deux peuples pouvant se retrouver avec leurs ressemblances autour des notions de patrie, de langue et de religion. ${ }^{8}$

7. Le 22 avril est désormais célébré par les Kurdes du monde entier comme la journée du journalisme.

8. Koç Y., « Kürdistan gazetesi... », 2013, p. 41. 
À la veille de la Première Guerre mondiale naît, sous l'impulsion des membres de l'organisation Hêvî [L'espoir] Rojî Kurd [Le soleil kurde]. Cette revue mensuelle connaîtra quatre numéros, dont le premier paraît le 6 juin 1913. Dirigée par Abdülkerim de Süleymaniye, qui étudiait à l'école militaire d'Istanbul (Harbiye) et devait devenir capitaine d'artillerie, la revue reçut de nombreuses contributions d'Abdullah Cevdet après qu'il eut abandonné le comité Union et Progrès (à l'inverse d'un Ziya Gökalp qui rejoignit les Jeunes Turcs après avoir publié le journal Peyman à Diyarbakır). Dans sa première livraison, la revue confie à la jeunesse une mission pour l'avenir et fait le serment de répondre, par les voies de la science et de la culture et en se gardant des ambitions politiques, aux injures faites à l'identité kurde. De fait, le contenu éditorial frappe par la place accordée aux travaux sur l'histoire et la langue kurdes. On y trouve en kurde les poèmes d'Ehmedê Xanî, Nalî (1798 - 1878) ou encore ceux du cheikh Rizayê Telebanî (1835 - 1910), ainsi qu'une polémique sur la question de l'alphabet, un certain nombre de contributeurs, dont Abdullah Cevdet, étant convaincus que l'alphabet arabe ne répond pas aux besoins de la langue kurde. Certains écrits valurent à leurs auteurs des séjours en prison, ces ennuis juridiques conduisant à l'interruption de la publication, qui reprendra immédiatement sous le titre Hetawî Kurd [La Lumière kurde] pour se poursuivre jusqu'à la déclaration de la Première Guerre mondiale.

Hêvî serait également à l'origine de la revue Yekbûn [L'Unité], dont le premier numéro parut en septembre 1913, incluant des articles en turc et en kurde. Mehmet Tayfun Malmisanij et Musa Anter relèvent que la revue met en exergue la nécessité d'alphabétiser les populations et pour cela de mettre en place un alphabet simple et facile. L'éditorial du troisième numéro indique que « les jeunes ne doivent pas se laisser emporter par l'ambition politique, leur plus grand honneur étant de participer à diverses organisations de façon à servir la nation. »

Les journaux et revues paraissant au lendemain de la Première Guerre mondiale placent eux aussi les enjeux du mouvement national kurde sur le terrain intellectuel, par opposition au champ politique. Ainsi de la revue Jîn [La Vie], qui paraît à Istanbul à l'automne 1918 et publie en turc et en kurde kurmanji ou sorani. Elle est d'abord dirigée par le fondateur de la Kürt Teali Cemiyeti [Association pour la valorisation des Kurdes], Müküslü Hamza, puis par Memduh Selim? . Jîn se propose de "restituer dans ses pages l'histoire, les droits nationaux, la sociologie et la littérature du peuple kurde ${ }^{10}$ et

9. Figure dont Mehmed Uzun retracera la vie dans son roman Siya Evînê.

10. Yücel M., Kürt Basın Tarihi..., 1998, p. 57-58. 
accorde une place importante aux poètes nationaux Ehmedê Xanî et Melayê Cizîrî mais aussi à la poésie et aux écrits de Piremêrd. On note parmi les contributeurs la présence de Kamuran Bedir Khan, Abdullah Cevdet ou encore Halil Heyali qui publie des articles liés à l'histoire, la mythologie, la sociologie, la linguistique ou la philosophie. Siverekli Hilmi transmet ses travaux sur les dictons et les expressions kurdes. Kemal Fevzî publie une étude sur l'origine des contes kurdes, qu'il relie à d'autres systèmes mythologiques, et donne à la revue trois nouvelles. Dès le deuxième numéro, Şefik Arvasî souligne la nécessité de développer l'apprentissage de la langue kurde pour libérer le peuple de l'ignorance et lui rendre sa dimension hérö̈que. Des appels répétés à l'unité aboutissent à la naissance, relayée dans le numéro 7 de la revue, d'une association : « Les préparatifs relatifs à la création d'une association scientifique du nom de Kürt Tamim-i Neşr-i Maarif Cemiyeti [Association pour la diffusion et la généralisation de la culture kurde], visant à publier des recherches sur la langue kurde, l'histoire et la géographie ainsi que sur l'économie et la sociologie, tout en diffusant les savoirs actuels parmi les Kurdes, sont maintenant achevés ${ }^{11} »$. Le numéro 22 , quant à lui, annonce la création d'une Kürt Kadınlar Teali Cemiyeti [Association pour la valorisation des femmes kurdes]. Les pressions du gouvernement finissent par avoir raison de la revue, qui livrera son dernier numéro le 2 octobre 1919 avant de renaître pour un temps sous forme de journal.

À la même époque, Mevlanzade Rıfat publie Serbestî, organe en turc de la Kürt Teali Cemiyeti. Les frères Celadet et Kamuran Bedir Khan, qui comptent alors pour les plus jeunes membres du mouvement kurde, en sont de fréquents collaborateurs. Entre le 9 novembre 1918 et le 21 mai 1919, Kamuran fait paraitre quatorze articles, Celadet huit, tous relatifs à la question kurde. ${ }^{12}$

11. Yücel M., Kürt Basın Tarihi..., 1998, p. 65.

12. Serfiraz M., "Kürt Basın Tarihine... », 2013, p. 37. Notons l'existence d'un certain nombre de titres pour lesquels une demande d'autorisation a été déposée, mais dont on ne sait s'ils ont ou non paru. Ainsi, Süreyya Bedir Khan a déposé le 6 novembre 1908 une demande d'autorisation pour Kürdistan, mais aussi pour un journal dénommé Aşiret. Müftizâde Reşid Efendi, de l'association Kürd Teavün ve Terakki Cemiyeti a fait la même démarche pour un journal dénommé Kürd en août 1908. Des démarches semblables ont été faites en dehors d'Istanbul pour d'autres titres : à Harput par Palutluzâde Mehmed Halil Efendi pour un hebdomadaire intitulé Füyûzât-ı Kürdiye, et à Van par David Papasyan pour İttihâd, un journal devant paraître en arménien, turc et kurde. On trouve enfin en 1910 une demande d'autorisation faite par Hüseyin Kenan Bedir Khan pour un journal intitulé Cudi, devant paraître en turc et en kurde. (Koç Y., "Kürdistan gazetesi... », 2013, p. 39-40). Nous avons évoqué ici des titres qui, outre le premier Kurdistan, étaient tous imprimés à Istanbul. On pourrait également mentionner des périodiques publiés à Diyarbakır : Peyman [L’accord] (1909), Amidi Sevda [Amed Passion] (1909), ou encore Gazî [L'appel] (1919). 


\section{La littérature en exil}

\section{Hawar}

Si les frères Bedir Khan sont aujourd'hui des figures prééminentes de l'histoire intellectuelle kurde, et à ce titre eux-mêmes devenus part de ce patrimoine intellectuel, c'est en premier lieu par leur travail autour de la revue Hawar [L'alarme], puis Ronahî [La lumière].

La chape de plomb qui s'abat sur le sud-est anatolien à partir de 1925 dans la foulée des révoltes du cheikh Said, de l'Ararat puis de Dersim, et dont l'une des principales conséquences en terme de répression est l'interdiction dans la région de l'usage de langues autres que le turc, amène Celadet Ali Bedir Khan et son frère Kamuran à poursuivre leurs activités linguistiques et littéraires en Syrie sous mandat français, aidés par les orientalistes Roger Lescaut, Pierre Rondot et Thomas Bois. Le premier numéro de Hawar sort en mai 1932. La revue, publiée en kurde et en français et qui connaîtra deux périodes de publication (1932-1935 et 1941-1943), se donne pour mission d'établir et diffuser un alphabet kurde basé sur l'alphabet romain et proche du nouvel alphabet turc pour en faciliter l'apprentissage, d'étudier l'histoire de la langue kurdes et ses différents dialectes, de publier le patrimoine oral des Kurdes : contes, légendes, chansons, mais aussi le patrimoine poétique écrit, d'en faire une classification chronologique accompagnée des biographies d'hommes de lettres et de personnalités illustres. Le patrimoine immatériel des Kurdes, dans sa dimension nationale, est au centre des préoccupations des contributeurs de la revue : il s'agit de faire l'étude de l'histoire et de la géographie du Kurdistan et de l'histoire des tribus, mais aussi d'étudier la musique et la danse en déterminant « les caractéristiques de la musique nationale, [en fixant et notant] ses mélodies ainsi que les caractéristiques, rythmes et figures des danses des Kurdes », ou encore les « mœurs et coutumes sociales, morales et religieuses du passé et du présent »; il s'agit enfin de " définir le costume national » et de déterminer les principes qui ont régi «la vie économique du pays, ses produits et son industrie nationale. " ${ }^{13}$ À côté du folklore, sont très vite présentés les poètes Ehmedê Xanî et Melayê Cizîrî. L'épopée Meme Alan quant à elle est publiée en feuilleton dès le quatrième numéro. Concernant la langue, ce travail de diffusion et de standardisation du kurmanji aura été pérenne, puisqu'il aboutira à la publication d'une grammaire qui fait aujourd'hui encore référence ${ }^{14}$.

13. Hawar, $\mathrm{n}^{\circ}$ 1, Damas, 1932.

14. Publiée progressivement à Damas entre 1941 et 1944 par Celadet Bedir Khan et Roger Lescot, elle sera achevée et publiée par Lescot en 1970 à Paris. 
Il convient de citer les principaux collaborateurs de Bedir Khan, Osman Sebrî, Cegerxwîn, Qedrî Cemîl Paşa et Qedrîcan, sans oublier Ehmedê Fermanê Kîkî, le dengbêj qui semble avoir été d'une aide si précieuse à Celadet au niveau de la collecte de la littérature orale.

La revue est publiée en kurde et en français. Pour le kurde, l'alphabet latin et l'alphabet arabo-persan sont utilisés conjointement jusqu'au numéro 23. À partir du numéro 24 seul l'alphabet latin est utilisé.

L'importance au niveau patrimonial de Hawar et du travail des frères Celadet et Karmuran est indéniable, visible et constamment affirmée, citée ou donnée en référence. Ainsi, en 2014, le premier numéro de la revue littéraire Wêje $\hat{u}$ Rexne s'ouvre, comme en guise de manifeste, sur un texte de Kamuran Alî Bedir Khan tiré des pages de Hawar et consacré à la littérature. Plus prosaïquement, le 15 mai, date du lancement de Hawar, est désormais commémoré comme jour de la langue kurde.

\section{Mehmed Uzun}

La littérature kurde contemporaine et l'approche des intellectuels kurdes contemporains vis-à-vis de la mémoire semblent placées sous la figure tutélaire de Mehmed Uzun, auteur de $1^{\prime}$ «école suédoise ${ }^{15}$ décédé en 2007, et dont l'ensemble du travail littéraire peut être considéré comme une tentative de sauvegarde du patrimoine immatériel des Kurdes. Écrire permet de sauver de l'oubli, la littérature est pour lui une forme, bien qu'imparfaite, de matérialisation de la mémoire.

À l'époque où commence l'aventure de Mehmed Uzun avec la littérature kurde, sa position intime vis-à-vis de la langue et du patrimoine culturel est marquée par un manque primordial qui se décline sous plusieurs aspects. Dépossession du langage tout d'abord, avec une relation marquée par l'interdit. La langue maternelle, la langue du foyer est interdite, rejetée par l'État sous la figure de l'instituteur et le traumatisme d'une gifle reçue pour un mot prononcé en kurde. Sa langue maternelle, c'est en 1972 et en prison qu'il commencera à l'apprendre, au contact d'intellectuels tels que Mehmet Emin Bozarslan ou Musa Anter. Il est arrêté une seconde fois le 21 mars 1976 en tant que rédacteur en chef de la revue Rizgari pour avoir publié des articles anonymes d'İsmail Beşikçi. Entendre, à son procès, le procureur nier l'existence de la langue kurde et des Kurdes eux-mêmes fait naître en lui un sentiment

15. Sur l'aventure intellectuelle de la diaspora kurde en Suède, on se réfèrera avec profit au travail de Clémence SCALbert-Yücel (2014). 
d'humiliation et d'impuissance qui suscitera sa décision de faire revivre la langue et l'histoire kurdes à travers la littérature. Alors que la poursuite de ses activités éditoriales menace de lui valoir une nouvelle condamnation, il décide de prendre la route de l'exil. Mais cette route qui doit, comme un certain nombre d'intellectuels kurdes, le conduire en Suède, passe d'abord par Damas.

Ce passage à Damas, tel qu'il le raconte dans un texte intitulé Welatê Xerîbiyêe : Bir Hüzündür Ayrllık [L'exil : la séparation est une tristesse], est l'occasion pour Mehmed Uzun de se plonger dans le passé bicentenaire des migrations kurdes. Il prend le temps d'y visiter les tombes des princes, poètes, écrivains, intellectuels et activistes kurdes qui y sont morts en exil : Bedir Khan Bey, Celadet Ali Bedir Khan, Qedrîcan ${ }^{16}$, Memduh Selim, Mevlana Halid. Un autre voyage en Syrie en 1986 le verra partir à la rencontre d'un maître dengbêj, Rıfatê Darê, qu'il écoutera réciter ses kılam [chansons] et l'épopée de Siyabend $\hat{u}$ Xecê pendant dix-sept jours afin de s'imprégner de sa langue et de récolter le vocabulaire nécessaire à son entreprise de création d'une langue romanesque kurde.

Le récit de cette rencontre prend place dans Dengbêjlerim ${ }^{17}$, recueil d'essais dans lequel Uzun est à la croisée des mémoires individuelle et collective. Le livre se veut à la fois un hommage et un acte de mémoire destiné à sauvegarder un vecteur, une forme en voie de disparation de la littérature populaire ${ }^{18}$, il fixe à la fois le souvenir d'Apê Qado, un dengbêj apparenté à sa famille, qu'il a connu enfant, et de certains des récits duquel il tire des réflexions sur son propre rapport à l'histoire et à la littérature, et d'autres dengbêj, comme Evdalê Zeynikêê ${ }^{19}$, qu'il n'a pas forcément connus ni entendus mais dont la renommée est ou a été notable chez les Kurdes. Dans le chapitre qu'il consacre

16. Abdulkadir (Kadri/Qedrî) Can [1911, Derik (Mardin), 1972] était un écrivain et poète dont les textes furent publiés dans les revues Hawar et Ronahî (il s'est exilé à Damas suite à la révolte du cheikh Said). Ses œuvres complètes ont été publiées par les éditions Belkî (le livre est aujourd'hui indisponible). Les éditions Lîs de Diyarbakır quant à elles ont édité en 2007 un recueil de nouvelles intitulé GUNEH [Le péché].

17. Que nous traduirions par «Mes Dengbêj » plutôt que par "Mes Bardes », le titre original procédant lui-même de l'association d'un vocable kurde et de la syntaxe turque, dans la visée transgressive de faire entrer un lexique kurde dans la langue turque, ce qui a lieu d'ailleurs à plusieurs reprises dans le texte lui-même. Un procédé également employé par d'autres auteurs d'origine kurde comme Murathan Mungan qui, dans Paranın Cinleri, fait entrer plusieurs mots kurdes dans la littérature turque.

18. Ce même souci de sauvegarde a suscité la création d'une " maison des Dengbêj » à Diyarbakır. 19. La vie de ce dengbêj a également inspiré à Uzun un roman : Rojek ji Rojên Evdalê Zeynikê [Un jour parmi les jours d'Evdalê Zeynikê], İstanbul, Orfeus, 1987. Il existe également un documentaire de Bülent Gündüz. 
à Evdalê Zeynikê, Uzun fait du dengbêj un résistant, détenteur du seul espace de liberté des Kurdes dans les périodes de coups d'état militaire.

« [...] Les années 1960 furent une nouvelle époque lugubre et silencieuse de junte militaire. On avait une fois encore, comme à chaque coup, fait passer des décrets contre les Kurdes. Une fois encore, être Kurde, parler kurde, agir en fonction de son identité kurde était devenu très difficile et très dangereux. Une fois encore les avenues, les rues, les lieux de divertissement s'étaient vidés, dans les maisons, loin des regards, plus que jamais, de petites assemblées s'étaient créées et les dengbêj avaient commencé à réciter, plus que jamais, de leurs voix brûlées, et à raconter la colère, la douleur, la tristesse, la séparation, l'exil, l'impuissance et l'oppression. Une fois encore, le dengbêj était devenu le seul refuge, la voix [deng] du dengbêj la seule consolation $[\ldots]^{20}$.»

Le dengbêj, vecteur oral du patrimoine, est un homme qui, par la prouesse de sa propre mémoire, a le pouvoir de sauvegarder, afin de la transmettre, la mémoire collective. Lorsqu'il sauve de l'oubli «ses » dengbêj en les faisant entrer dans la littérature, c'est, autant que la mémoire de cet art, lui-même en voie de disparition, celle que cet art s'est donné pour mission de véhiculer qu'il cherche à préserver et à fixer dans une matérialité, d'abord celle de l'enregistrement sonore (qui n'est cependant pour lui qu'un outil), puis celle de l'écrit. Le romancier assume alors une fonction similaire à celle d'une institution patrimoniale telle que la Mala Dengbêjan de Diyarbakır (maison des dengbêj), qui cherche à perpétuer la tradition tout en menant un travail d'archivage.

Et au-delà de son utilité, en tant que réserve de vocables et de contes, pour le romancier qu'il cherche à devenir, le dengbêj devient une figure à laquelle il s'identifie à travers la langue telle qu'il en a vu se manifester les pouvoirs en la personne d'Alihan, compagnon de cellule taciturne qui, parvenu à l'extrême limite du désespoir, s'est claustré dans une récitation continuelle de l'épopée de Siyabend.

«[...] Oui, avant toute chose il y avait la voix. La voix devint verbe; le verbe, expression harmonieuse ; l'expression, le récit mesuré ; le récit, histoire ; et l'histoire identité, culture et civilisation. Le verbe a créé la langue ; la langue le monde, la connaissance du monde, son commentaire. (...) Le verbe a créé la mémoire et lui a permis de couler comme un fleuve à travers les époques et l'histoire humaine $[\ldots]^{21}$ »

Mehmed Uzun semble donc s'être donné pour mission de devenir un dengbêj de l'écrit, et donc un agent de restitution et de protection d'un patrimoine

20. Mehmed Uzun, Dengbêjlerim. Nous nous référons à la réédition d'Ithaki (Istanbul) de 2006, p. 34 et sv.

21. Op. cit., p. 114-115. 
littéraire, le dernier maillon d'une chaîne initiée par Homère et à laquelle il a laissé se raccrocher Ehmedê Fermanê Kîkî, le dernier personnage de Dengbêjlerim, compagnon de travail de Celadet Ali Bedirhan en exil.

La mémoire, concept sur lequel il s'appesantit dans ce dernier chapitre, est sans conteste au centre de ses préoccupations de romancier. Si tous ont une motivation mémorielle, certains de ses romans semblent avoir pour ambition d'intégrer au processus de renaissance de la littérature kurde les figures essentielles de l'histoire kurde du xx siècle. Le dengbêj Evdalê Zeynikê dans Rojek ji Rojên Evdale Zeynikê, Memduh Selim dans Siya Evînê [La poursuite de l'ombre], Celadet Ali Bedir Khan dans Bîra Qederê [Le puits du destin] et son aïeul, l'Émir Bedir Khan dans Hawara Dîcleyê [L'appel du tigre].

Cette fonction mémorielle du roman kurde dans sa première période était ressentie par Uzun comme essentielle, vitale, pour un peuple dépossédé de sa mémoire, de sa langue, de son histoire. Autant pour survivre au présent que pour être en mesure d'écrire l'avenir :

«[...] La mémoire - et pour quelqu'un dont la mémoire a été atrophiée, affaiblie, réinventée, distordue, l'importance de la mémoire; il faut se jeter aux trousses du temps passé, tout évoquer, se souvenir de tout. Le passé doit être empêché de n'être que le passé. Pour que la douleur, l'affliction, la mélancolie du présent soient transformées en forces créatrices, il faut faire du passé le temps vécu aujourd'hui et il faut le vivre très intensément. Il faut retrouver les pistes effacées, les voix disparues et les visages qui sont restés derrière la fenêtre brouillée du temps et il faut les rendre à la vie. Il faut revenir aux racines, au commencement, au principe. Il faut retrouver cette sobriété sans péché du commencement, du premier instant, cette simplicité sans calcul. Pas seulement pour savoir ou se souvenir pour savoir, il faut retrouver cela pour $\mathrm{s}^{\prime}$ en souvenir continûment, il faut s'en souvenir sans cesse $[\ldots]^{22} »$.

Il semble cependant désormais que, sept ans après la disparition de Mehmed Uzun et alors que la solidité de sa relève ne peut être mise en doute (Arjen Arî, Firat Cewerî, Helîm Yûsiv, Lal Laleş, Şener Özmen ou Ciwanmerd Kulek ${ }^{23}$ pour ne citer que quelques noms parmi une pléthore d'auteurs, romanciers, nouvellistes, poètes ou dramaturges actuellement en activité), la littérature

\footnotetext{
22. Dengbêjlerim, p. 114 .

23. Cet écrivain, auteur de quatre romans, construit également une œuvre de traducteur. II traduit de l'espagnol ou de l'anglais vers le kurde des classiques de la littérature mondiale tels que Faulkner, Joyce ou Marquez et voit dans la traduction non seulement un moyen de transmission mais également l'opportunité de contribuer au renforcement et à la standardisation de la langue cible (voir son intervention au salon du livre de Londres en 2013 : http://www.youtube.com/ watch?v=r2dofi2Augg).
} 
kurde contemporaine soit prête à s'affirmer comme une littérature nationale à part entière, qui s'appuie sur un patrimoine autant que sur des parcours individuels soucieux d'expérimentation et de modernité.

Cette production s'appuie sur quelques maisons d'édition dont les plus remarquables s'emploient à lancer et développer la littérature contemporaine tout en conservant dans leurs catalogues, pour les transmettre avec une visée de patrimonialisation, les classiques. Şeyhmus Diken, écrivain-essayiste, acteur de la société civile et conseiller à la municipalité HDP de Diyarbakır sur les questions de mémoire, nous l'affirme clairement : ces classiques doivent rester disponibles à tout moment. Ce qui explique que de multiples éditions des œuvres d'Ehmedê Xanî apparaissent simultanément chez plusieurs éditeurs : les éditions Nûbihar, créées en 1992 à Istanbul par Süleyman Çevik, éditent à côté d'une édition en kurde une édition bilingue (kurde/turc) de Mem $\hat{u}$ Zîn ; les éditions Avesta, fondées en 1996 à Istanbul par Abdullah Keskin, disposent de plusieurs éditions du même titre : une en kurde moderne, une en turc, une en anglais, disponibles, pour les deux premières, en édition reliée à côté des versions courantes brochées, et ce dans le cadre d'une collection d'œuvres complètes baptisée « Ehmedê Xanî Külliyatı ». Ces œuvres complètes sont également éditées en un volume par les éditions Lîs, fondées à Diyarbakır en 2004 et dirigées par le poète Lal Laleş, dans le cadre d'une collection de «Kürt Klasikleri » qui pour l'instant ne comporte pas d'autres titres.

Ce qui ne signifie pas que l'entreprise de patrimonialisation des éditions Lîs se cantonne à Ehmedê Xanî. Lal Lalêş et son équipe ont également lancé une collection baptisée «Pirtûkxaneya [Bibliothèque] Ehmedê Xanî ». Cette figure tutélaire, qui marque la volonté d'intégrer au patrimoine littéraire des auteurs plus proches dans le temps, veille sur une vingtaine d'ouvrages (en kurde) d'auteurs du xx siècle et plus particulièrement de la période « Hawar ». On y retrouve ainsi des textes d'Ereb Şemo (écrivain ézidi d'Arménie, considéré comme l'auteur du premier roman kurde, Le berger kurde), Didim et Hopo, de Qedrî Can, Guneh, d'Osman Sebrî, Li Goristaneke Amedê, de Nureddin Zaza, Gulê, de Cegerxwîn, Reşoyê Darê, Cîm ûu Gulperî, de Kamuran Ali BedirKhan, Eyloyê Pîr, et de Celadet Ali Bedir-Khan, Klêopatre, Hevind et Gazinda Xencere Min.

Cette patrimonialisation de la figure des Bedir-Khan est également accomplie par les éditions Avesta qui disposent d'une collection d'œuvres complètes pour chacun des deux frères. Quatre livres de Kamuran y sont publiés, dont un manuel de lecture kurde (Xwendina Kurdî), mais aussi les œuvres littéraires de Celadet et surtout ses travaux linguistiques : son ouvrage sur l'alphabet kurde, sa grammaire du kurde kurmanji élaborée avec Roger Lescot et son dictionnaire unilingue, ces deux derniers livres faisant également l'objet 
d'une double édition, reliée et brochée, ces éditions de luxe marquant, comme pour Mem $\hat{u}$ Zîn, l'importance d'une œuvre à conserver. Cette volonté de patrimonialisation des auteurs de la période « Hawar » se retrouve également chez Avesta dans la collection des œuvres complètes de Cegerxwîn (dix volumes).

Ces deux exemples, Avesta et Lîs, nous semblent remarquables car le processus de transmission qui est à la base de toute entreprise de patrimonialisation y est particulièrement visible, l'édition de ces textes littéraires du passé accompagnant une politique d'ouverture très nette sur une littérature contemporaine en cours de construction.

\section{Conclusion}

La tenue du cinquième salon du livre de Diyarbakır en mai 2014 était l'occasion de se rendre compte, autant par le nombre d'éditeurs présents que par la diversité des conférences organisées en marge, que la situation, vingt ans après la parution du premier roman en kurde de Mehmed Uzun, est celle d'un véritable bouillonnement rendu possible et visible par l'ouverture politique vis-à-vis des Kurdes et de la langue kurde en Turquie d'une part, d'autre part par l'accession à sa nécessaire maturité de ce mouvement centenaire que des générations successives d'auteurs ont réussi à mener à bien. 11 faudrait cependant que ce mouvement soit pérenne, et pour cela notamment que l'enseignement du kurde progresse de manière significative de façon à développer le lectorat potentiel. Dans un deuxième temps, cette littérature devra dépasser ses limites régionales et communautaires en appelant à un véritable mouvement de traduction (la politique bilinguiste des éditions Doğan, qui publie les traductions kurdes de livres en turc d'écrivains kurdes comme Yavuz Ekinci ${ }^{24}$ ou Murat Özyaşar ${ }^{25}$, bien que louable, reste limitée), d'une part vers le turc, mais aussi et si possible sans tarder dans les langues qui pourront commencer à ouvrir à la littérature kurde sa place dans la littérature mondiale et la faire rayonner hors de ses frontières présentes. Mais ceci demande, outre l'intérêt des éditeurs étrangers, que le temps et les acteurs

24. Parus chez Doğan Kitap, d'abord en turc, puis en kurde : Cennetin Kayıp Toprakları (2012) / Erden Bihuşte Yen Winda (2013) [Les terres disparues du paradis], Tene Yazılan Ayetler (2010) / Ayeten Li Can Nıvisandi (2011) [Des versets écrits sur la peau].

25. Ayna Çarpması [Le coup du miroir] (2008) / Bîr [Le puits] (2011). 
concernés, traducteurs en premier lieu, fassent leur travail de sélection pour que ressortent de cette profusion éditoriale les œuvres qui témoigneront, demain, de cette vitalité actuelle. Vitalité qui, loin de se cantonner à la littérature, s'exprime dans d'autres domaines : ainsi, cette année les fruits du projet BAK, Se Souvenir et Raconter, axé sur la jeune création photo/vidéo dans les villes de Diyarbakır, Batman, İzmir et Çanakkale ont été présentés au public. Le théâtre aussi semble être un espace en pleine effervescence, à la croisée de la mémoire et du présent, autant à Batman qu'à Diyarbakır dont le théâtre municipal s'efforce constamment de programmer des textes en kurde. Il organise depuis 2012 un concours d'écriture de pièces de théâtre où la problématique mémorielle s'est naturellement invitée puisque l'on trouve, par exemple, parmi les lauréats de la première édition, une œuvre consacrée à Evdalê Zeynikê. Le théâtre et les dengbêj constituent encore l'argument du projet DÎWAN, un atelier de recherche sur le jeu d'acteur organisé à Diyarbakır en septembre 2014 par le réalisateur et comédien Mîrza Metîn et qui "évolue entre l'ancien et le nouveau, en tentant de se souvenir de l'ancien sans pour autant oublier le nouveau », cherchant ainsi " un lieu entre le jeu d'acteur occidental et l'art du conteur oriental » à travers l'analyse des « techniques du mouvement, de la voix et du conte » sur un axe basé sur l'art des dengbêjj, çiro$k b e ̂ j$ et des recherches sur le folklore. On voit ainsi comment ne cessent de dialoguer l'ancien et le nouveau, le passé et le présent, ou comment s'articulent à travers la littérature ou les arts le travail de mémoire et l'affirmation de l'identité et comment, dans un contexte où être locuteur ne signifie pas nécessairement être lecteur, le théâtre devient un vecteur complémentaire à celui de l'édition dans sa mission de transmission d'un patrimoine.

\section{Bibliographie}

Bedir Khan Emir Djeladet \& Lescot Roger, Grammaire kurde (dialecte kurmanji), Paris, Librairie d'Amérique et d'Orient, 1970.

Blau Joyce, Mémoire du Kurdistan, Paris, Findakly, 1984.

Bozarslan Hamit, «Le kémalisme et le problème kurde », in Halkawt Hakim (ed.), Les Kurdes par-delà l'exode, Paris, L'Harmattan, 1992.

Bozarslan Mehmet Emîn, Jîn Kovara Kurdî-Tirkî 1918-1919 [Revue La Vie, kurde-turc], Upsala, Deng Yay., 1985-1988, 5 vols.

Celil Celilê, Kürt Aydınlanması [Le mouvement d'intellectualisation des Kurdes], İstanbul, Avesta, 2001. 


\section{Reconquêtes patrimoniales, réaffirmations identitaires}

Cemal Hasan, «Bir Portre : Mehmed Uzun » [Un portrait : Mehmed Uzun], Milliyet, 17.11. 2006.

DıкEN Şeyhmus, Zevalsiz Ömrün Sürgünü Mehmed Uzun [Mehmed Uzun, perpétuel exilé de la vie], Diyarbakır, Lîs, 2009.

GöкçEN Amed, Ezidiler. Kara Kitap Kara Talih [Les Ézidis, Livre noir, noire fortune], İstanbul, Bilgi Üniversitesi Yayınları, 2014.

Hawar, Damas, 1932-1935 et 1941-1943.

Kaya Fetullah, Osmanlı Döneminde Kürt Basın [La presse kurde dans la période ottomane], Yüksek Lisans Tezi, İstanbul, Marmara Üniversitesi, 2008.

Koç Yener, « Kürdistan Gazetesi (1908-1909) üzerine birkaç not » [Quelques notes sur le journal Kurdistan], Kürt Tarihi, n 8, août-septembre 2013.

Kulek Ciwanmerd, Defterên Perrîdankan [Les cahiers des papillons], İstanbul, Avesta, 2014.

Lescot Roger (ed.), Mamé Alan. Épopée kurde, Paris, Gallimard, 1999 [1942].

Malmisanij Mehmet Tayfun, Kürt Talebe Hevi Cemiyeti, İlk Legal Kürt Öğrenci Derneği [L'association des étudiants Kurdes Hevi. La première association légale d'étudiants kurdes], İstanbul, Avesta Yayınları, 2002.

Meretowar A., Amedi C., Aslan S. Azad, Rojî Kurd. Kovara Cemiyeta Hêvî [Rojî Kurd, la revue de l'association Hêvî], İstanbul, War yayınları, 2002.

Metîn Mîrza, Jêrzemîn, Şanoya li Jêrzemînê, di navbera salên 1991 û 2013 an de li Tirkiyê Şanoya Kurdî [Underground, Le théâtre Underground, Le théâtre kurde en Turquie de 1991 à 2013], Diyarbakır Büyükşehir Belediyesi, 2014.

Mungan Murathan, Paranın Cinleri [Les djinns de l'argent], İstanbul, Metis, 1997.

Nezan Kendal, « Préface », in Lescot R. (ed.), Mamé Alan. Épopée kurde, Paris, Gallimard, 1999 [1942].

Nikitine Basile, Les Kurdes. Étude sociologique et historique, Paris, Klincksieck, 1956.

SCALBERT-YüCEL Clémence, Engagement, langue et littérature. Le champ littéraire kurde en Turquie (1980-2000), Paris, éditions Petra, 2014.

SECCADî Alaeddin, Mêjûwî̀ edeb̂̀ Kurdî [Anthologie de la littérature kurde], Bagdad, 1952.

Serfiraz Mesûd, « Kürt Basın Tarihine genel bir bakış » [Un tour d'horizon de l'histoire de la presse kurde], Kürt Tarihi, n 8, août-septembre 2013.

SunAr Erhan, Veda Oyunu [La pièce aux adieux], İstanbul, Alakarga, 2014.

ŞEmo Ereb, Şivanê Kurd, édition russe en 1931, édition kurde non datée à Beyrouth, édition bilingue kurde-français par l'Institut kurde de Paris, 1989.

Tejel Gorgas Jordi, Le mouvement kurde de Turquie en exil. Continuités et discontinuités du nationalisme kurde sous le mandat français en Syrie et au Liban (1925-1946), Berne, Peter Lang, 2007. 


\section{La littérature comme instrument de sauvegarde du patrimoine culturel}

Temo Selim, Kürt Şiiri Antolojisi [Anthologie de la poésie kurde], İstanbul, Agora Kitaplı̆̆ 2007.

Uzun Mehmed, Tĥu [Toi], Stockholm, Dengê Komal, 1984.

Uzun Mehmed, Rojek ji Rojên Evdalê Zeynikê [Un jour parmi les jours d'Evdalê Zeynikê], İstanbul, Orfeus, 1987.

Uzun Mehmed, Siya Evînê, İstanbul, Doz Yayınları, 1992. Fawaz Hussain (trad.), La poursuite de l'ombre, Paris, Phébus, 1999.

Uzun Mehmed, Destpekâ Edebiyate Kurdî [Introduction à la littérature kurde], Ankara, Beybûn, 1992.

Uzun Mehmed, Antolojia Edebiyata Kurdî [Anthologie de la littérature kurde], İstanbul, Tüm Zamanlar Yayıncılık, 1995.

Uzun Mehmed, Bîra Qederê [Les puits du destin], Stockholm, Sara, İstanbul, Avesta, 1995.

Uzun Mehmed, «Welatê Xerîbiyê : Bir Hüzündür Ayrılık » [L'exil : la séparation est une tristesse], Nar Çiçekleri [Fleurs de grenade], Ithaki, 1996.

Uzun Mehmed, Dengbêjlerim [Mes dengbêj], İstanbul, Belge Yayınları, 1998.

Uzun Mehmed, Hawara Dîcleyê 1 [L'appel du tigre], İstanbul, Avesta, 2001, Hawara Dîcleyê 2, İstanbul, Avesta, 2003.

Wêje û Rexne [Littérature et critique], Diyarbakır, N 1, janvier-avril 2014.

Yekemîn Pêşbaziya Nivîsandina Lîstikên Şanoya Kurdî [Premier concours d'écriture de pièces de théâtre en kurde], Diyarbakır, Weşanen Şaredariya Bajarê Mezin a Amedê, 2012 ; Duyemîn Pêşbaziya Nivîsandina Lîstikên Şanoya Kurdî [Recueil des lauréats de la seconde édition du concours], même lieu, [2015].

YüCEl Müslüm, Kürt Basın Tarihi (Tekzip) [Histoire de la presse kurde], İstanbul, Aram Yayınları, 1998. 